\title{
Long-Term Outcome of Patients with Antiphospholipid Syndrome Who Undergo Percutaneous Coronary Intervention
}

\author{
Leor Perl Adi Netzer Eldad Rechavia Tamir Bental Abid Assali Pablo Codner \\ Aviv Mager Alexander Battler Ran Kornowski Eli I. Lev \\ Department of Cardiology, Rabin Medical Center, Petach Tikva, and the Sackler Faculty of Medicine, \\ Tel Aviv University, Tel Aviv, Israel
}

\section{Key Words}

Antiphospholipid antibody syndrome $\cdot$ Percutaneous coronary intervention $\cdot$ Long-term outcome

\begin{abstract}
Objectives: Patients with antiphospholipid antibody syndrome (APS) have an increased risk of atherothrombotic complications. There are limited data regarding the outcome of patients with APS who undergo percutaneous coronary intervention $(\mathrm{PCl})$. Accordingly, we aimed to assess the long-term outcomes of these patients. Methods: Nineteen APS patients who underwent PCI between the years 2003 and 2008 were compared to 380 patients who had undergone $\mathrm{PCl}$ during the same period ( $\mathrm{PCl}$ group) and were matched by age ( \pm 5 years), gender, diabetes and hypertension. In addition, APS patients were compared to 1,458 patients with ST segment elevation myocardial infarction (MI) who were treated with $\mathrm{PCl}$ during the same period. Sixmonth to 4-year clinical outcomes were evaluated. Results: The indication for PCl in the APS group was acute coronary syndrome in $52.6 \%$ of patients. After 1 year of follow-up, patients with APS had higher rates of target vessel revascularization than the other two groups, which translated to higher rates of major adverse cardiac events. There were no differences in $\mathrm{Ml}$ or mortality rates between the groups.
\end{abstract}

Conclusions: Patients with APS who undergo PCI have worse long-term clinical outcomes, driven by higher rates of revascularization, than other patients undergoing $\mathrm{PCl}$. Further study is warranted to examine the mechanisms underlying these findings.

Copyright $\odot 2012$ S. Karger AG, Basel

\section{Introduction}

Antiphospholipid antibody syndrome (APS) is an entity comprising both clinical and laboratory features. Based on the most recent criteria, the definition of APS requires at least one clinical and one laboratory manifestation, i.e. vascular thrombosis (arterial, venous or small vessel thrombosis), pregnancy-related morbidity (an unexplained fetal death after the 10th week of gestation, 3 or more unexplained consecutive spontaneous abortions before the 10th week of gestation or premature birth of a morphologically normal neonate before the 34 th week of gestation due to eclampsia or severe preeclampsia) and laboratory parameters, detected at least twice, 12 weeks apart (anticardiolipin, anti- $\beta_{2}$-glycoprotein I or lupus anticoagulant) [1].

APS is the most common acquired form of blood protein defect, resulting in both arterial and venous throm-

\section{KARGER}

Fax +4161306 1234

E-Mail karger@karger.ch

www.karger.com
(C) 2012 S. Karger AG, Basel

$0008-6312 / 12 / 1222-0076 \$ 38.00 / 0$

Accessible online at:

www.karger.com/crd
Leor Perl, MD

Rabin Medical Center

The Sackler Faculty of Medicine, Tel Aviv University

39 Jabotinsky St., Petah-Tikva 49100 (Israel)

Tel.+972 3937 2251, E-Mail leorperl@gmail.com, leorper@clalit.org.il 
botic events [2]. Patients with APS have an increased risk of atherothrombotic complications, such as cerebrovascular events and myocardial infarction (MI) [3-6]. Both cerebrovascular and coronary artery diseases are more prevalent in patients with APS and comprise the major cause of mortality and morbidity in these patients $[7,8]$. The presence of APS is a strong risk factor for the development of coronary artery disease, especially in young patients [9]. In the Risk of Arterial Thrombosis In Relation to Oral Contraceptives study, the odds ratio for an $\mathrm{MI}$ in young women was 5.3 times higher in patients with lupus anticoagulant as compared to other women [10]. The increased risk of premature atherosclerosis and its thrombotic complications is probably caused by proinflammatory and procoagulant effects on vascular endothelial cells, or indirectly via immunoinflammatory mechanisms underlying autoantibody-mediated thrombosis [11-15].

Although the syndrome is known for its vascular complications, many of the cardiovascular aspects of APS remain largely obscure. In particular, there is a paucity of data regarding the outcome of patients with APS who undergo coronary revascularization. Small studies have demonstrated that patients with APS are predisposed to high rates of restenosis of the coronary arteries and vascular grafts after percutaneous coronary intervention (PCI) or coronary artery bypass graft surgery, respectively, causing significant morbidity and mortality [16-19]. Some of these studies were conducted more than a decade ago, in the percutaneous transluminal coronary angioplasty era, and are therefore less relevant for contemporary PCI practice [16, 19]. Other studies did not define and focus on APS patients as a specific study group, but rather included patients with certain antibodies considered part of the syndrome. Also, some of these studies included only patients with secondary APS, such as in the case of APS and systemic lupus erythematosus (SLE). These studies cannot fully shed light on the prognosis of the unique group of patients with APS, both primary and secondary, after revascularization. Therefore, given the paucity of data, we aimed to assess the long-term outcomes of patients with APS who undergo PCI.

\section{Methods}

The Rabin Medical Center PCI registry was systematically reviewed for patients with a diagnosis of APS who underwent PCI between the years 2003 and 2008. Patients with APS were identified according to the diagnoses listed in their charts. In all cases, we verified that the diagnosis of APS met with current criteria [1]. Clinical manifestations, such as vascular thrombosis or pregnancy-related morbidity, were identified. In addition, two separate positive antiphospholipid antibodies (aPL; anticardiolipin IgG and/or IgM, anti- $\beta_{2}$-glycoprotein I IgG and/or IgM or lupus anticoagulant) were required for APS diagnosis and inclusion in the study. After careful selection, 19 patients with confirmed APS were identified. Sixteen of them were classified as primary APS, and 3 were diagnosed with SLE, and therefore had secondary APS. For each patient with APS, 20 control non-APS patients who underwent PCI during the same period were matched by age $( \pm 5$ years), gender, diabetes mellitus and hypertension.

The control group was identified from the Rabin Medical Center PCI registry, which is a comprehensive database which processes all the data regarding all consecutive cases of PCI from 2003 and onwards performed at the two hospitals of the Rabin Medical Center. It includes all the procedural data, as well as clinical, laboratory, echocardiographic and subsequent event data, from several sources, i.e. the patients' electronic medical record system, the medical centers' demographic information system, the medical centers' data warehouse and the medical centers' central laboratory database. Data are periodically integrated, reviewed, checked for accuracy and analyzed. Data collection was approved by the hospital ethics committee in compliance with the Declaration of Helsinki, with waiver for the need of individual informed consent.

Since the majority of the patients in the APS group had undergone PCI because of an acute coronary syndrome (ACS), and in order to enable comparison to a high-risk group, a secondary analysis was performed in which the APS group was compared to patients with ST segment elevation MI (STEMI) who were treated with PCI. For this purpose, we used the Rabin Medical Center's STEMI primary PCI registry, which is a prospective registry of consecutive patients with STEMI who underwent primary PCI at the Rabin Medical Center between the years 2003 and 2008. The registry includes demographic, clinical, angiographic and procedural data. Immediate and in-hospital events are recorded from the hospital charts. For each patient, a standardized questionnaire is completed either by telephone or in the outpatient clinic at 6 months and yearly thereafter. A total of 1,458 patients with STEMI were included in the present study as the second control group (after excluding the APS patients and patients with cardiogenic shock).

For all groups, accuracy of the mortality data was verified with the Israel Central Bureau of Statistics, Interior Ministry of Israel. All data regarding patients' prior and subsequent hospitalizations, including all ICD-9 diagnoses, were retrieved from the medical centers' data warehouse. Repeated revascularization procedures and episodes of MI were confirmed using the hospital and affiliated hospital databases.

All patients (in all groups) were treated with aspirin before the PCI and clopidogrel (300-600 mg) either before PCI (pretreatment) or immediately after the procedure. Unfractionated heparin (70 U/kg loading) was given before PCI, and the dosage was adjusted to achieve an activated clotting time of 200-250 s during the intervention. Glycoprotein IIb/IIIa receptor inhibition by eptifibatide was used at the discretion of the operator. All patients were prescribed lifelong aspirin and clopidogrel $(75 \mathrm{mg} /$ day $)$ for 3-12 months, depending on stent type.

Acute STEMI was defined as the presence of typical chest pain and accompanying symptoms for $\geq 30 \mathrm{~min}$ but $<12 \mathrm{~h}$ in the pres- 
Table 1. Baseline clinical characteristics in APS versus PCI groups

\begin{tabular}{lrll}
\hline Parameter & $\begin{array}{l}\text { APS } \\
(\mathrm{n}=19)\end{array}$ & $\begin{array}{l}\text { PCI } \\
(\mathrm{n}=380)\end{array}$ & $\begin{array}{l}\mathrm{p} \\
\text { value }\end{array}$ \\
\hline Age, years & $60.2 \pm 11.8$ & $60.1 \pm 9.0$ & 1 \\
Women & $8(42.1)$ & $163(42.9)$ & 1 \\
Hypertension & $16(84.2)$ & $300(78.9)$ & 0.6 \\
Diabetes & $6(31.6)$ & $125(32.9)$ & 0.9 \\
Hyperlipidemia $^{1}$ & $17(89.5)$ & $113(29.7)$ & $<0.0001$ \\
Current/past smoking $_{\text {Chronic renal failure }}^{2}$ & $7(36.8)$ & $186(48.9)$ & 0.3 \\
Prior coronary artery bypass $_{\quad}$ surgery & $2(10.5)$ & $33(8.7)$ & 0.8 \\
Prior left ventricular dysfunction & $7(36.8)$ & $43(11.3)$ & 0.005 \\
\hline
\end{tabular}

Figures in parentheses are percentages.

${ }^{1}$ Diagnosis previously made by physician, or patient receiving lipid-lowering therapy.

${ }^{2}$ Creatinine levels $>1.5 \mathrm{mg} / \mathrm{dl}$ or creatinine clearance $<60 \mathrm{ml} /$ $\mathrm{min} / \mathrm{m}^{2}$.

Table 2. Baseline clinical characteristics in APS versus STEMI groups

\begin{tabular}{lccl}
\hline Parameter & $\begin{array}{l}\text { APS } \\
(\mathrm{n}=19)\end{array}$ & $\begin{array}{l}\text { STEMI } \\
(\mathrm{n}=1,458)\end{array}$ & $\begin{array}{l}\mathrm{p} \\
\text { value }\end{array}$ \\
\hline Age, years & $60.2 \pm 11.8$ & $60.7 \pm 13$ & 0.2 \\
Women & $8(42.1)$ & $289(19.8)$ & 0.005 \\
Body mass index & $27.2 \pm 3$ & $28.3 \pm 4$ & 0.8 \\
Hypertension & $16(84.2)$ & $694(47.6)$ & 0.002 \\
Diabetes & $6(31.6)$ & $375(25.7)$ & 0.6 \\
Hyperlipidemia ${ }^{1}$ & $17(89.5)$ & $685(47.0)$ & 0.0002 \\
Current or past smoking & $7(36.8)$ & $540(37.0)$ & 1 \\
Chronic renal failure ${ }^{2}$ & $2(10.5)$ & $104(7.2)$ & 0.6 \\
Prior coronary artery bypass & & & \\
$\quad$ surgery & $3(15.8)$ & $39(2.7)$ & 0.02 \\
Prior stroke & $5(26.3)$ & $110(7.5)$ & 0.01 \\
Prior MI & $3(15.8)$ & $133(9.1)$ & 0.3 \\
Baseline medications & & & \\
$\quad$ Aspirin & $16(84.2)$ & $1,264(86.7)$ & 0.7 \\
$\quad$ Clopidogrel & $8(42.1)$ & $672(46.1)$ & 0.7 \\
$\quad$ Statins & $15(78.9)$ & $445(30.5)$ & 0.0001 \\
$\quad$ B-Blockers & $2(10.5)$ & $149(10.2)$ & 0.95 \\
$\quad$ ACE inhibitors/ARB & $4(21.1)$ & $162(11.1)$ & 0.2 \\
\hline
\end{tabular}

Figures in parentheses are percentages. $\mathrm{ACE}=$ Angiotensinconverting enzyme; $\mathrm{ARB}=$ angiotensin receptor blockers.

${ }^{1}$ Diagnosis previously made by physician, or patient receiving lipid-lowering therapy.

${ }^{2}$ Creatinine levels $>1.5 \mathrm{mg} / \mathrm{dl}$ or creatinine clearance $<60 \mathrm{ml} /$ $\min / \mathrm{m}^{2}$. ence of ST segment elevation $\geq 1 \mathrm{~mm}$ in $\geq 2$ contiguous leads or new or undetermined duration of left branch bundle block in association with $\geq 2$-fold increase in cardiac enzymes (troponin I or T). The diagnosis of (re-)infarction during follow-up was based on recurrent chest pain suggestive of an acute MI, accompanied by a repeated increase in cardiac enzymes to $\geq 2$ times the upper limit of normal $\geq 48 \mathrm{~h}$ after PCI and/or new ST elevation or pathologic Q wave on surface electrocardiography. Target vessel revascularization (TVR) was defined as any revascularization that involved the target vessel. Major adverse cardiac events (MACE) included any of the following (without repetition): death, nonfatal MI or TVR. All events were further adjudicated by a research coordinator and reviewed by an experienced cardiologist from our research team. Follow-up was completed for $100 \%$ of the APS patients at all stages. The PCI group's follow-up rates were $95 \%$ at 6 months, $89 \%$ at 1 year, $72 \%$ at 2 years and $61 \%$ at 3 years. Follow-up rates for the STEMI group were 100, 93, 84 and 75\%, respectively, at the corresponding time periods.

Data are presented as means \pm standard deviation. Continuous variables were compared using Student's t test. Categorical variables were compared using $\chi^{2}$ statistics or Fisher's exact test, as appropriate. All tests were two-tailed, and a p value $<0.05$ was considered significant. Survival analysis in the APS versus PCI analysis was performed using life table analysis and a Cox proportional hazards procedure with covariates as follows: APS, diabetes mellitus, hypertension, previously known heart failure, previously known moderate-to-severe left ventricular dysfunction, smoking status and baseline creatinine. Statistical analysis was performed using SPSS version 16 (SPSS, Chicago, Ill., USA).

\section{Results}

Clinical characteristics of the APS group and the two control groups are presented in tables 1 and 2. Because of disparities in clinical characteristics, matching was performed according to gender, age, diabetes and hypertension status, with a ratio of 1:20 between the APS patients and the PCI group (table 1). The only discrepancies that remained after matching were higher rates of left ventricular dysfunction and hyperlipidemia in patients with APS (table 1). Patients with APS were also compared to the STEMI group in secondary analysis (table 2). In this latter comparison, patients with APS were more likely to be women, have hypertension and hyperlipidemia and be treated with statins than patients with STEMI. In addition, they had higher rates of stroke and coronary artery bypass surgery in the past. The indication for PCI among the patients with APS was an ACS in 10 patients (52.6\%, including $31.6 \%$ with acute MI and $21 \%$ with recent MI or unstable angina). The rest of the patients were treated for stable angina. All patients with APS were treated with Coumadin (warfarin) before and after the PCI. However, the international normalized ratio (INR) at admission was above 1.5 in only $37 \%$ of the patients. 
Table 3. Angiographic characteristics and indications for the index procedure

\begin{tabular}{lccc}
\hline & $\begin{array}{l}\text { APS } \\
(\mathrm{n}=19)\end{array}$ & $\begin{array}{l}\text { PCI } \\
(\mathrm{n}=380)\end{array}$ & $\begin{array}{l}\text { STEMI } \\
(\mathrm{n}=1,458)\end{array}$ \\
\hline ACS & $10^{*}(52.6)$ & $200(52.6)$ & $1,458(100)$ \\
One-vessel disease & $5(26.3)$ & $91(23.9)$ & $581(39.8)$ \\
Two-vessel disease & $6(31.6)$ & $134(35.3)$ & $502(34.4)$ \\
Three-vessel disease & $8(42.1)$ & $154(40.5)$ & $375(25.7)$ \\
$\begin{array}{l}\text { Intervention to proximal } \\
\quad \text { left anterior descending }\end{array}$ & & & \\
$\quad$ artery & $4(21.1)$ & $89(23.4)$ & $506(34.7)$ \\
$\begin{array}{l}\text { Use of drug-eluting stents } \\
\text { Mean stent diameter, mm }\end{array}$ & $7(36.8)$ & $157(41.3)$ & $390(26.7)$ \\
Total stent length, mm & $26.9 \pm 15$ & $3.0 \pm 0.3 \pm 20$ & $3.1 \pm 0.5$ \\
\hline
\end{tabular}

Figures in parentheses are percentages. ${ }^{*} \mathrm{p}<0.0001$ for acute coronary syndrome rate between APS versus STEMI groups; all other comparisons between APS and the other two groups were nonsignificant (PCI and STEMI groups were not compared with each other).

Eight patients with APS (42.1\%) received clopidogrel before the PCI (mostly because of history of stroke), and they all continued to receive long-term clopidogrel treatment after the procedure. The other patients in the group generally received clopidogrel for 3-12 months after the PCI, depending on the presentation (ACS or stable angina) and the type of stent implanted during the procedure. In the PCI group, patients generally received clopidogrel for 3-12 months, depending on stent type, and in the STEMI group, the majority of the patients received clopidogrel for 1 year following the PCI. All patients received long-term aspirin after the procedure. Angiographic and procedural characteristics of the index procedure were similar between the 2 groups (table 3 ), including the frequency of the left anterior descending artery as the culprit vessel, the presence of multivessel disease, stent characteristics and infrequent use of drugeluting stents.

Clinical outcomes of the groups are presented in tables 4 and 5. Data are presented with reference to the followup rates at the beginning of each period. Patient follow-up reached 4 years (fig. 1). As compared to the general PCI group, patients with APS had higher rates of TVR, beginning at 6 months of follow-up and onwards, which translated to higher rates of MACE, a difference that had become significant after 1 year. The groups did not differ with regard to rates of MI or mortality (fig. 1; table 4). Data are shown both according to outcomes in each time
Table 4. Clinical outcomes in the APS versus PCI groups

\begin{tabular}{lccl}
\hline Outcome & APS $(\mathrm{n}=19)$ & PCI $(\mathrm{n}=380)$ & $\mathrm{p}$ value \\
\hline 6 Months & & $\mathrm{n}=360$ & \\
Death & $5.3 \%$ & $4.2 \%$ & 0.6 \\
TVR & $15.8 \%$ & $3.4 \%$ & 0.03 \\
MACE & $21.1 \%$ & $7.8 \%$ & 0.07 \\
\hline 1 Year & & $\mathrm{n}=339$ & \\
Death & $10.5 \%$ & $5.9 \%$ & 0.3 \\
TVR & $26.3 \%$ & $5.3 \%$ & 0.005 \\
MACE & $36.8 \%$ & $11.5 \%$ & 0.006 \\
\hline Years & & $\mathrm{n}=275$ & \\
Death & $10.5 \%$ & $8.4 \%$ & 0.2 \\
TVR & $31.6 \%$ & $6.9 \%$ & 0.003 \\
MACE & $47.4 \%$ & $15.3 \%$ & 0.002 \\
\hline 3 Years & & $\mathrm{n}=232$ & \\
Death & $10.5 \%$ & $9.4 \%$ & 0.4 \\
TVR & $42.1 \%$ & $7.8 \%$ & 0.0002 \\
MACE & $52.6 \%$ & $18.1 \%$ & 0.002 \\
\hline
\end{tabular}

Table 5. Clinical outcomes in the APS versus STEMI groups

\begin{tabular}{lccl}
\hline Outcome & $\begin{array}{l}\text { APS } \\
(\mathrm{n}=19)\end{array}$ & $\begin{array}{l}\text { STEMI } \\
(\mathrm{n}=1,458)\end{array}$ & $\mathrm{p}$ value \\
\hline months & \multicolumn{3}{c}{$\mathrm{n}=1,458$} \\
MI & $10.5 \%$ & $5.2 \%$ & 0.3 \\
TVR & $15.8 \%$ & $9.0 \%$ & 0.3 \\
Death & $5.3 \%$ & $5.1 \%$ & 1 \\
MACE & $21.1 \%$ & $14.4 \%$ & 0.3 \\
\hline year & & $\mathrm{n}=1,356$ & \\
MI & $10.5 \%$ & $6.5 \%$ & 0.4 \\
TVR & $26.3 \%$ & $11.2 \%$ & 0.03 \\
Death & $10.5 \%$ & $6.2 \%$ & 0.1 \\
MACE & $36.8 \%$ & $18.5 \%$ & 0.04 \\
\hline years & \multicolumn{3}{c}{$\mathrm{n}=1,225$} \\
MI & $15.8 \%$ & $7.6 \%$ & 0.2 \\
TVR & $31.6 \%$ & $13.8 \%$ & 0.03 \\
Death & $10.5 \%$ & $7.8 \%$ & 0.4 \\
MACE & $47.4 \%$ & $22.4 \%$ & 0.01 \\
\hline 3 years & \multicolumn{3}{c}{$\mathrm{n}=1,094$} \\
MI & $15.8 \%$ & $8.5 \%$ & 0.2 \\
TVR & $42.1 \%$ & $15.6 \%$ & 0.002 \\
Death & $10.5 \%$ & $9.4 \%$ & 0.7 \\
MACE & $52.6 \%$ & $26.8 \%$ & 0.01 \\
\hline
\end{tabular}


Fig. 1. Cumulative event rates of all-cause mortality (a), death or MI (b), TVR (c) and MACE (death, MI or TVR; d) in patients with APS $(n=19)$ versus matched patients who underwent PCI $(\mathrm{n}=380)$. From 6 months and onwards, patients with APS had higher rates of TVR, which translated into higher rates of MACE. NS = not significant.

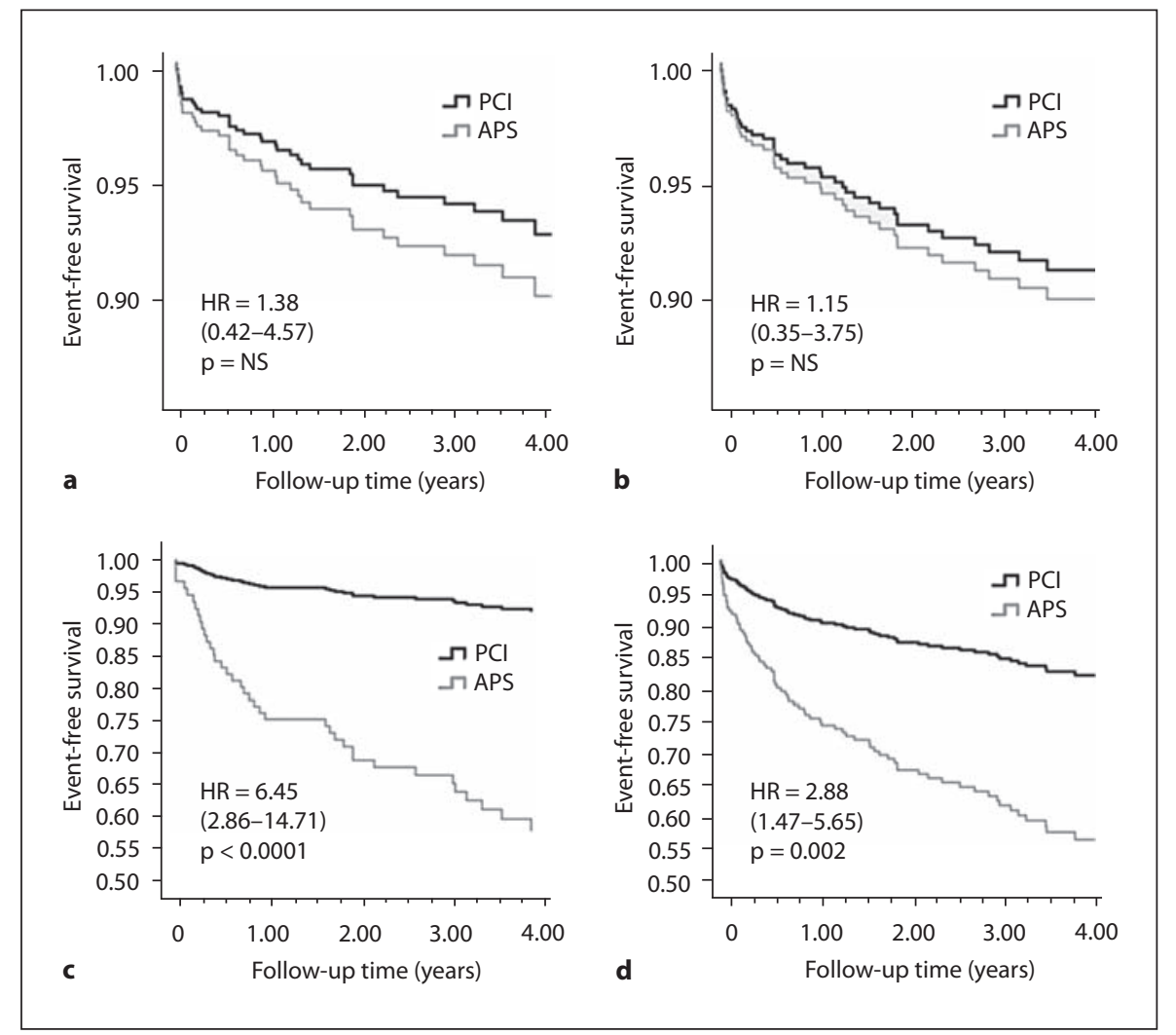

period, based on analysis of categorical variables (table 4), and as Kaplan-Meier curves, derived from life table analysis methods, as described above (fig. 1). Patients with APS also had higher rates of TVR and MACE when compared to patients with STEMI (table 5). These differences became apparent after 1 year and were maintained afterwards. The two groups did not differ with regard to the rates of MI or mortality. Matching of the STEMI group to the APS group according to parameters of age, hyperlipidemia, hypertension and diabetes status did not change these results (data not shown).

\section{Discussion}

The current study assessed the long-term outcomes of patients with APS who had undergone PCI. Patients with APS were compared to a matched group of patients without APS who underwent PCI, and also to a high-risk group of patients with STEMI treated by PCI. We found that patients with APS had higher long-term rates of adverse cardiac events when compared to both the general PCI and the STEMI groups. This difference was driven by exceedingly high rates of revascularization in the APS group. Unexpectedly, ischemic complications did not differ between the groups.

The most striking finding of our study are the remarkably high rates of TVR in patients with APS who have undergone PCI, despite similar use of drug-eluting stents and similar diabetes status as the control groups. These revascularization procedures were probably caused by both restenosis within the stent(s) and accelerated atherosclerosis in vessel areas not covered by stents.

An increased risk of restenosis in patients with APS who undergo PCI has been reported previously in a few small studies. Gürlek et al. [18] reported a strong association between the levels of anticardiolipin antibodies and the outcome of PCI after an ACS. In this study, higher levels of anticardiolipin antibodies predicted increased rates of restenosis after PCI, without an effect on mortality. Similarly, in the percutaneous transluminal coronary angioplasty era, Eber et al. [19] reported higher rates of restenosis after PCI (with balloon angioplasty) in patients with higher levels of anticardiolipin. The presence of APS also appears to be associated with an increased risk of vein graft disease after coronary artery bypass graft. In 
fact, the risk for accelerated vein graft disease and failure has been shown to correlate well with the levels of anticardiolipin antibodies [20,21]. Thus, our findings, taken together with previous reports, indicate that the presence of APS is a strong risk factor for the development of stent restenosis as well as vein graft disease. Evidence also exists in regards to enhanced atherosclerosis progression and premature ischemic heart disease in patients with APS. Ames et al. [22] reported that levels of IgG anticardiolipin independently predict the carotid artery intimamedia thickness. Moreover, previous reports have linked levels of anticardiolipin with both the risk and extent of peripheral vascular disease $[23,24]$ and the risk of MI and cardiac death [25].

The potential causes for increased restenosis rates and accelerated atherosclerosis in patients with APS are numerous. A direct pathophysiological effect of aPL on coronary artery endothelium has been suggested. Inhibition of endothelial cell production of prostacyclin may cause endothelial dysfunction as well as enhanced platelet aggregation [26]. Another suggested mechanism may include immune complexes formed by aPL autoantibodies, which may deposit in the vessel wall, causing inflammation and wall injury. In addition, $\beta_{2}$-glycoprotein I antibodies are known to activate and damage endothelial cells [27]. These processes all lead to endothelial dysfunction as well as a thrombotic diathesis [26-28]. Secondly, anticardiolipin antibodies can potentially cross-react with antibodies against oxidized low-density lipoprotein (LDL), due to similarities between the cardiolipin and $\beta_{2^{-}}$ glycoprotein I and LDL molecules [29]. In turn, antibodies against oxidized LDL have been clearly linked to accelerated atherosclerosis progression [30]. Thirdly, aPL may potentially promote local intimal smooth muscle cell proliferation and restenosis indirectly, by enhancing the release of cytokines and growth factors [31-33]. Finally, medical treatment and other systemic comorbidities in patients with APS may enhance atherosclerosis and restenosis indirectly, such as in the case of steroid treatment and renal disease in SLE patients. However, in our study group only 3 patients were diagnosed with SLE, and only 1 patient had received steroids at admission and on a regular basis.

Despite the known propensity for thrombotic complications in patients with APS, as well as case reports of recurrent stent thrombosis [34-36], we did not observe differences in the rates of MI or death between the APS and the control groups. This may have been caused by lack of statistical power, as the group of patients with APS was relatively small. Also, it is possible there was a selec-

Long-Term Outcome of PCI in APS Patients tion bias in the previously diagnosed APS group, since all patients were well treated and under regular medical surveillance before (and after) the index procedure. Most importantly, all the patients with APS were treated with anticoagulation (vitamin $\mathrm{K}$ antagonists) before and after the PCI, in addition to antiplatelet therapy. However, as pointed out, while all APS patients were prescribed vitamin K antagonists by their physicians, it seems that compliance rates for therapy were low, as attested by the nontherapeutic INR levels at admission in many of the patients (63\%). It is possible that these low rates of therapeutic INR levels on admission may have contributed to the ACS presentation in some of the patients.

The present study has several limitations. Firstly, the APS group is of relatively small size and was compared to a large group of patients, limiting the statistical power of the study. Secondly, there were significant imbalances between the groups, mainly with regard to clinical characteristics. However, following the matching process to the PCI group, most imbalances were resolved. Thirdly, the study was based on a registry, with all the limitations inherent to a nonrandomized trial. Fourthly, we did not have data regarding the exact duration of clopidogrel therapy following PCI in all patients. Finally, our study group mostly comprised patients with primary APS but also included 3 patients with secondary APS. Mechanisms of coronary disease may differ between these two groups. For instance, SLE patients with APS have been shown to have a higher rate of atherosclerotic plaque formation when assessed for intima-media thickness. In primary APS, a causative association between the presence of aPL antibodies and accelerated atherosclerosis is yet to be established $[37,38]$. The association between aPL and the increased rates of TVR in our study may be explained by a pathological effect of aPL on the endothelium, leading to endothelial dysfunction and perturbation, and through the induction of a more inflammatory local environment (via cytokines and growth factors), thus overall increasing the risk for restenosis [38].

Despite these limitations, our study highlights the increased risk patients with APS have for long-term complications after PCI and, in particular, the need for revascularization. The clinical implications of this study, as well as of previous reports, are that an early and aggressive approach to the treatment of patients with APS undergoing coronary intervention is warranted. Such an approach may include the administration of high-dose statins and antiplatelet and anticoagulant therapy. However, treatment of these patients before and after PCI poses several therapeutic dilemmas, such as the duration of 
dual antiplatelet therapy in addition to anticoagulation and the choice of bare metal versus drug-eluting stents, balancing the risks of thrombosis and restenosis versus bleeding. Future studies may help guide the therapeutic strategy. In addition, further research examining the mechanisms which underlie the increased rates of restenosis and revascularization in patients with APS is warranted.

\section{References}

$\checkmark 1$ Miyakis S, Lockshin MD, Atsumi T, et al: International consensus statement on an update of the classification criteria for definite antiphospholipid syndrome (APS). J Thromb Haemost 2006;4:295-306.

2 Bick RL: Antiphospholipid thrombosis syndromes. Hematol Oncol Clin North Am 2003; 17:115-147.

-3 Cervera R, Piette JC, Font J, et al; Euro-Phospholipid Project Group: Antiphospholipid syndrome: clinical and immunologic manifestations and patterns of disease expression in a cohort of 1,000 patients. Arthritis Rheum 2002;46:1019-1027.

4 Koniari I, Siminelakis SN, Baikoussis NG, et al: Antiphospholipid syndrome; its implication in cardiovascular diseases: a review. J Cardiothorac Surg 2010;5:101.

5 Kaplan SD, Chartash EK, Pizzarello RA, et al: Cardiac manifestations of the antiphospholipid syndrome. Am Heart J 1992;124: 1331-1338.

6 Soltész P, Szekanecz Z, Kiss E, et al: Cardiac manifestations in antiphospholipid syndrome. Autoimmun Rev 2007;6:379-386.

-7 Sacré K, Brihaye B, Hyafil F, et al: Asymptomatic myocardial ischemic disease in antiphospholipid syndrome: a controlled cardiac magnetic resonance imaging study. Arthritis Rheum 2010;62:2093-2100.

8 Ruiz-Irastorza G, Crowther M, Branch W, et al: Antiphospholipid syndrome. Lancet 2010;376:1498-1509.

-9 Takeuchi S, Obayashi T, Toyama J, et al: Primary antiphospholipid syndrome with acute myocardial infarction recanalised by PTCA. Heart 1998;79:96-98.

10 Urbanus RT, Siegerink B, Roest M, et al: Antiphospholipid antibodies and risk of myocardial infarction and ischaemic stroke in young women in the RATIO study: a casecontrol study. Lancet Neurol 2009;8:9981005.

11 Ames PR, Margarita A, Sokoll KB, et al: Premature atherosclerosis in primary antiphospholipid syndrome: preliminary data. Ann Rheum Dis 2005;64:315-317.

12 Medina G, Casaos D, Jara LJ, et al: Increased carotid artery intima-media thickness may be associated with stroke in primary antiphospholipid syndrome. Ann Rheum Dis 2003;62:607-610.

13 Ames PR, Antinolfi I, Scenna G, et al: Atherosclerosis in thrombotic primary antiphospholipid syndrome. J Thromb Haemost 2009;7:537-542.
Sherer Y, Zinger H, Shoenfeld Y, et al: Atherosclerosis in systemic lupus erythematosus. Autoimmunity 2010;43:98-102.

15 Long BR, Leya F: The role of antiphospholipid syndrome in cardiovascular disease. Hematol Oncol Clin North Am 2008;22:7994.

16 Ludia C, Domenico P, Monia C, et al: Antiphospholipid antibodies: a new risk factor for restenosis after percutaneous transluminal coronary angioplasty? Autoimmunity 1998;27:141-148.

17 Maksimowicz-McKinnon K, Selzer F, Manzi $S$, et al: Poor 1-year outcomes after percutaneous coronary interventions in systemic lupus erythematosus: report from the National Heart, Lung, and Blood Institute Dynamic Registry. Circ Cardiovasc Interv 2008;1:201208.

18 Gürlek A, Ozdöl C, Pamir G, et al: Association between anticardiolipin antibodies and recurrent cardiac events in patients with acute coronary syndrome. Int Heart J 2005; 46:631-638.

19 Eber B, Schumacher M, Auer-Grumbach P, et al: Increased IgM-anticardiolipin antibodies in patients with restenosis after percutaneous transluminal coronary angioplasty. Am J Cardiol 1992;69:1255-1258.

20 Bick RL, Ismail Y, Baker WF Jr: Coagulation abnormalities in patients with precocious coronary artery thrombosis and patients failing coronary artery bypass grafting and percutaneous transcoronary angioplasty. Semin Thromb Hemost 1993;19:412-417.

21 Morton KE, Gavaghan TP, Krilis SA, et al: Coronary artery bypass graft failure - an autoimmune phenomenon? Lancet 1986;2: 1353-1357.

22 Ames PR, Margarita A, Delgado Alves J, et al: Anticardiolipin antibody titre and plasma homocysteine level independently predict intima media thickness of carotid arteries in subjects with idiopathic antiphospholipid antibodies. Lupus 2002;11:208-214.

23 Glueck CJ, Lang JE, Tracy T, et al: Evidence that anticardiolipin antibodies are independent risk factors for atherosclerotic vascular disease. Am J Cardiol 1999;83:1490-1494.

24 Nityanand S, Bergmark C, de Faire U, et al: Antibodies against endothelial cells and cardiolipin in young patients with peripheral atherosclerotic disease. J Intern Med 1995; 238:437-443.

25 Vaarala $\mathrm{O}$, Mänttäri M, Manninen V, et al: Anti-cardiolipin antibodies and risk of myo- cardial infarction in a prospective cohort of middle-aged men. Circulation 1995;91:2327.

26 Mackworth-Young C: Antiphospholipid antibodies: more than just a disease marker? Immunol Today 1990;11:60-65.

27 Meroni PL, Riboldi P: Pathogenic mechanisms mediating antiphospholipid syndrome. Curr Opin Rheumatol 2001;13:377-382.

28 Roubey RA: Mechanisms of autoantibodymediated thrombosis. Lupus 1998;7(suppl 2):S114-S119.

29 Vaarala O, Alfthan G, Jauhiainen M, et al: Crossreaction between antibodies to oxidised low-density lipoprotein and to cardiolipin in systemic lupus erythematosus. Lancet 1993;341:923-925.

30 Salonen JT, Ylä-Herttuala S, Yamamoto R, et al: Autoantibody against oxidised LDL and progression of carotid atherosclerosis. Lancet 1992;339:883-887.

31 Libby P, Schwartz D, Brogi E, et al: A cascade model for restenosis. A special case of atherosclerosis progression. Circulation 1992; 86(6 suppl):III47-III52.

32 Aikawa M, Sakomura Y, Ueda M, et al: Redifferentiation of smooth muscle cells after coronary angioplasty determined via myosin heavy chain expression. Circulation 1997;96: 82-90.

33 Jara LJ, Medina G, Vera-Lastra O: Systemic antiphospholipid syndrome and atherosclerosis. Clin Rev Allergy Immunol 2007;32: 172-177.

34 Muir DF, Stevens A, Napier-Hemy RO, et al: Recurrent stent thrombosis associated with lupus anticoagulant due to renal cell carcinoma. Int J Cardiovasc Intervent 2003;5:44-46.

35 Weissman A, Coplan NL: Antiphospholipid antibody syndrome and acute stent thrombosis. Rev Cardiovasc Med 2006;7:244-246.

36 Middlebrooks EH, Panda M: Multiple recurrent stent thrombosis in a patient with coexisting clopidogrel resistance and increased anticardiolipin antibodies: a case report. Case Report Med 2010;2010:974149.

37 Ames PR, Margarita A, Alves JD: Antiphospholipid antibodies and atherosclerosis: insights from systemic lupus erythematosus and primary antiphospholipid syndrome. Clin Rev Allergy Immunol 2009;37:29-35.

38 Gualtierotti R, Biggioggero M, Meroni PL: Cutting-edge issues in coronary disease and the primary antiphospholipid syndrome. Clin Rev Allergy Immunol 2011, E-pub ahead of print. 